\title{
Percepción de las pacientes con cáncer de mama sobre aspectos de ayuda en psicoterapia de grupo
}

\author{
Olga Herrero', Eva Rodríguez, Luis Botella, Sergi Corbella, \\ Antonia María Gómez y Meritxell Pacheco \\ Universidad Ramón Llull, Asociación Española contra el Cáncer - Junta de Barcelona
}

\section{RESUMEN}

Los grupos de psicoterapia constituyen una de las modalidades de intervención más eficaces para la mejora de la calidad de vida de las mujeres con cáncer de mama. Pero, además de los contenidos, en una dinámica grupal se dan otro tipo de procesos que pueden influir en la eficacia de la intervención. El objetivo del presente estudio es conocer más a fondo los microprocesos de ayuda y no ayuda según la percepción de las propias pacientes. Los principales resultados de este estudio con una muestra de 50 pacientes muestran que los aspectos de ayuda percibidos como más significativos tienen que ver con los componentes relacionales del propio grupo así como con el establecimiento de una buena alianza terapéutica, mientras que la percepción de no ayuda se asocia sobretodo a la excesiva individualidad y objetivos no compartidos. Los resultados obtenidos permiten también señalar otros factores relevantes para una intervención eficaz.

Palabras clave: Psicoterapia de grupo, aspectos de ayuda, cáncer de mama.

\begin{abstract}
Psychotherapy groups represent one of the most effective types of intervention for improving the quality of life of women with breast cancer. However, apart from the content of group sessions themselves, other types of events related to the group dynamics can influence on the outcome of the intervention. The aim of this study is to gain a deeper knowledge of the meaningful and non-meaningful events based on the opinions of patients. The main results obtained from a sample of 50 patients show that the most significant aspects of meaningful events observed are to do with the relationships formed within the group itself, together with a good therapeutic support, whilst the perception of non meaningful events is mostly associated with an excessive individuality and unshared goals. The results obtained also point to other relevant factors to achieve an effective intervention.
\end{abstract}

Keywords: Psychotherapy group, meaningful events, breast cancer. 


\section{Introducción}

El cáncer es uno de los principales problemas de salud en nuestro entorno y una de las principales causas de muerte prematura en los países desarrollados. De los diferentes tipos de tumores, uno de los que más relevancia ha tenido y continua teniendo debido a su frecuencia y a las dificultades que genera su diagnóstico y tratamiento, es el cáncer de mama.

En la Unión Europea, se diagnostican aproximadamente 275.000 casos nuevos (29\%) y la mortalidad se sitúa en torno a los 130.000 (17,3\%). La mayoría de los casos se presentan en mujeres postmenopáusicas, y la media de edad en el momento del diagnóstico es alrededor de 60 años. La probabilidad de desarrollar un cáncer de mama antes de los 75 años es del 8\% (Boyle y Ferlay, 2004; Parkin, Whelan, Ferlay y Storm, 2005).

En todos los registros españoles, el cáncer de mama es el tumor más frecuente en mujeres, se diagnostican aproximadamente 16.000 casos nuevos al año (más del 25\% de los casos de cáncer). Mueren unas 5.971 mujeres anualmente (19\%), y la media de edad de fallecimiento es de 66,5 años (Cabanes, Pérez-Gómez, Aragonés, Pollán y López-Abente, 2009; Curado, Edwards, Storm y Boyle, 2007; Fundación Científica de la Asociación Española contra el Cáncer, 2005; Parkin et al., 2005).

Los programas de detección precoz junto con los avances diagnósticos y terapéuticos se han traducido en un incremento de la supervivencia, estos factores han contribuido a que en España la supervivencia a los 5 años de las mujeres afectadas sea del 80,3\%, similar a la media europea, 79,4\% (Cabanes et al., 2009;
Sant, Allemani, Santaquilani, Knijn, Marchesi y Capocaccia, 2009). Se estima que actualmente en España existen unas 70.000 mujeres diagnosticadas de cáncer de mama en los últimos 5 años, que suponen el $34 \%$ de los casos prevalentes de cáncer en mujeres en nuestro país (Ferlay, Bray, Pisani y Parkin, 2004).

En Cataluña, el cáncer de mama es el tumor de mayor incidencia entre la población femenina. Actualmente se diagnostican aproximadamente 4.675 casos nuevos cada año, esta incidencia se situaría por debajo de la media europea y por encima de la media española. Anualmente mueren más de 1.000 mujeres catalanas por esta causa (19\%). Actualmente la supervivencia es del 78,48\%, y aproximadamente 46.150 mujeres conviven con un diagnóstico de cáncer de mama (Institut Català d'Oncologia, 2001a, 2001b; Izquierdo, Gispert, Saladie y Espinàs, 2008).

Los datos presentados anteriormente sitúan al cáncer de mama como uno de los problemas de salud más frecuentes en nuestra sociedad y que, debido a su historia natural de larga evolución y las posibilidades de recaída y de enfermedad avanzada, permiten considerarla como una enfermedad crónica. Situación que comporta que muchas personas tengan que convivir con la enfermedad durante muchos años, enfrentándose a una serie de dificultades, tanto a nivel físico como emocional, que pueden dejar vivir este tipo de experiencia y otras que pueden aparecer a lo largo del tiempo, disminuyendo la calidad de vida de algunas mujeres con cáncer de mama (Bower et al., 2006; Helgeson y Tomich, 2005; Michael, Berkman, Colditz, Holmes y Kawachi, 2002; Stanton, 2006). 
El hecho de haber superado un cáncer de mama y haber finalizado todos los tratamientos oncológicos necesarios, no implica necesariamente que la mujer se recupere totalmente física y emocionalmente de la experiencia. Aunque muchos supervivientes de cáncer presentan una buena adaptación, más del $40 \%$ presentan síntomas clínicamente relevantes de malestar psicológico. Posiblemente la disminución en el funcionamiento familiar y social, incluyendo problemas de pareja; disminución en la participación de actividades lúdicas; pérdida de interés por la actividad sexual y un peor funcionamiento sexual, son las consecuencias negativas más significativas a largo plazo del diagnóstico de cáncer (Lemieux, Bordeleau y Goodwin, 2007; Schnoll, Knowles y Harlow, 2002).

Aún así, revisiones como la de Mols, Vingerhoets, Coebergh y Van de Poll-Franse (2005) sobre la calidad de vida a largo plazo de pacientes supervivientes de cáncer de mama llegan a la conclusión de que las mujeres supervivientes experimentan una buena calidad de vida global. Por tanto, podemos afirmar que, a corto plazo, las mujeres que han sobrevivido a un cáncer presentan dificultades emocionales (Bower et al., 2006; Stanton, 2006; Tartaro, Roberts, Nosarti, Crayford, Luecken y David, 2005) y que, cuando comparamos la calidad de vida en aquellas pacientes en las que ha pasado más tiempo desde el diagnóstico de la enfermedad con pacientes con menos años de supervivencia, la calidad de vida es mayor en las primeras así como también es mayor el bienestar emocional y social en el primer grupo de pacientes (Neyt y Albrech, 2006; Helgeson y Tomich, 2005).
Las mujeres con cáncer de mama supervivientes presentan una serie de necesidades que, en ocasiones, independientemente del tiempo transcurrido desde el diagnóstico de la enfermedad, no están cubiertas. En un estudio realizado por Hodgkinson, Butow, Hunt, Pendlebury, Hobbs y Wain (2007) con 117 mujeres supervivientes de cáncer de mama con tiempos desde el diagnóstico entre 2-10 años, los autores encontraron que las pacientes manifestaron ansiedad y necesidades de apoyo (86\%), principalmente relacionadas con cuestiones existenciales como tratar las preocupaciones sobre la recidiva del cáncer (manifestada por una tercera parte de las supervivientes) y necesidades informativas. Resultados que también se confirman en otros estudios realizados con supervivientes de cáncer de mama (Ferrell, Grant, Funk, OtisGreen y García, 1998; Rosedale y KrainovichMiller, 2008; Vivar y McQueen, 2005).

Concretamente, el diagnóstico de cáncer de mama comporta muchas y variadas reacciones emocionales, como pueden ser de shock, incredulidad, miedo, ansiedad, depresión, pérdida de control, disminución de la autoestima, dificultades con las relaciones personales y familiares, problemas de pareja, etc., en otras palabras, queda alterada la calidad de vida de las pacientes (Avis, Crawford y Manuel, 2004; Carlsson y Hamrin, 1994; Michael et al., 2002; Pugliese, Fabi, Maggi, Falcicchio y Cognetti, 2006; Salvatierra, 1995; Schnoll et al., 2002).

Ante estas reacciones emocionales, en muchas ocasiones, la paciente diagnosticada de cáncer de mama necesita apoyo y orientación psicológica para afrontar de la 
mejor manera posible todas las dificultades y obstáculos que se va encontrando en esta nueva situación, que desborda las propias capacidades y recursos. La psicoterapia puede mejorar el malestar emocional que la mayoría de personas experimentan cuando se les diagnostica la enfermedad y por las secuelas derivadas de los tratamientos, mejorando la calidad de vida y facilitando el proceso de adaptación a la enfermedad, mejoría que se mantiene al año de haber finalizado la intervención (Bellver, 2007; Cruzado y Olivares, 1996; Font, 2003; García-Lumbreras y Blasco, 2008; Given et al., 2004; Hogan, Linden y Najarian, 2002; Lindemalm, Strang y Lekander, 2005; McArdle et al., 1996; Moorey et al., 1994; Rehse y Pukrop, 2003; Sebastián, Bueno, Mateos y García, 1999; Yoo, Ahn, S. B. Kim, Kim y Han, 2005).

La intervención psicológica difiere en objetivos y técnicas y puede ser más o menos compleja, en función de las dificultades concretas que afrontan los pacientes, las cuales pueden estar asociadas con el tipo de enfermedad, su estadio y los tratamientos oncológicos recibidos. Las diferentes modalidades de psicoterapia en oncología, incluyen estrategias como facilitar información, educación para la salud, proporcionar habilidades de afrontamiento adecuadas, ventilación de emociones, corregir pensamientos distorsionados, enseñar técnicas de control de la ansiedad como relajación, visualización, etc. (Cimprich, Janz, Northouse, Wren, Given, B. y Given, C., 2005; Fawzy, Fawzy, Arndt y Pasnau, 1995; Gielissen, Verhagen, Witjes y Bleijenberg, 2006; Larson, Duberstein, Talbot, Caldwell y Moynihan, 2000; Molassiotis,
Yung, Yam, Chan y Mok, 2002; Okamura, Fukui, Nagasaka, Koike y Uchitomi, 2003; Roffe, Schmidt y Ernst, 2005).

Estas posibilidades de tratamiento se pueden realizar de forma individual (McArdle et al., 1996; Moorey, Greer, Bliss y Law, 1998; Wilson, Taliaferro y Jacobsen, 2006), en formato de grupo (Cameron, Booth, Schlatter, Ziginskas y Harman, 2007; Greenstein y Breitbart, 2000; Kissane et al., 2003; Sebastián et al., 1999) o en pareja (Baider, 1995; Bultz, Speca, Brasher, Geggie y Page, 2000; Kuijer, Buunk, de Jong, Ybema y Sanderman, 2004).

Posiblemente los mecanismos psicológicos que explican la eficacia de estas intervenciones psicológicas son mejorar la autoeficacia, la sensación de control y las interpretaciones de los acontecimientos (Andersen, 1992; Bárez, 2002).

En revisiones realizadas por Fawzy (1999) y Font (2003), ambos autores coinciden en las recomendaciones que establecen sobre las intervenciones psicológicas más adecuadas, en función de las demandas físicas y emocionales que requiere cada fase de la enfermedad oncológica. En la Tabla 1 se muestran las recomendaciones realizadas por Font (2003).

Fawzy (1999) concluye que estas intervenciones son útiles cuando se utilizan individualmente, pero son más potentes y eficaces cuando se utilizan de forma combinada. Y Font (2003) concluye que aunque no parece que exista un tipo de intervención claramente eficaz para ayudar a los pacientes oncológicos en su adaptación a la enfermedad, todas ellas muestran efectos beneficiosos en la mejora de la calidad de vida. 
Tabla 1

Principales objetivos de la intervención psicológica en cáncer (Font, 2003, p. 61)

\begin{tabular}{|c|c|c|}
\hline TIPO & OBJETIVOS PRINCIPALES & FASE ENFERMEDAD \\
\hline Educativo-informativa & $\begin{array}{l}\text { Mejorar la información y la } \\
\text { participación de los pacientes } \\
\text { en la toma de decisiones, fa- } \\
\text { cilitar la aceptación del diag- } \\
\text { nóstico }\end{array}$ & Diagnóstico \\
\hline Terapia conductual & $\begin{array}{l}\text { Control de síntomas, mejora } \\
\text { del afrontamiento conductual }\end{array}$ & $\begin{array}{l}\text { Tratamiento inicial, comple- } \\
\text { mentario y recidiva }\end{array}$ \\
\hline Psicoterapia individual & $\begin{array}{l}\text { Facilitar la adaptación emo- } \\
\text { cional y la expresión/ discu- } \\
\text { sión de aspectos personales y } \\
\text { vivenciales }\end{array}$ & $\begin{array}{l}\text { Recidiva, enfermedad avan- } \\
\text { zada }\end{array}$ \\
\hline Psicoterapia de grupo & $\begin{array}{l}\text { Facilitar la comunicación y el } \\
\text { aprendizaje vicario de estra- } \\
\text { tegias, apoyo social, facilitar la } \\
\text { adaptación a la vida cotidiana }\end{array}$ & $\begin{array}{l}\text { Intervalo libre, enfermedad } \\
\text { avanzada }\end{array}$ \\
\hline
\end{tabular}

En palabras de Font (2003): "Sí que parece que las intervenciones tipo counselling son el procedimiento a seguir ante problemas informativos y de comunicación, especialmente en la fase de diagnóstico; las intervenciones cognitivo-conductuales para mejorar el afrontamiento de los síntomas, efectos secundarios y problemas concretos de la vida cotidiana (fase de tratamiento); la terapia no directiva para los problemas emocionales y las preocupaciones interpersonales (fase de intervalo libre); y la psicoterapia para las necesidades de reflexión existencial sobre la propia vida y los nuevos valores (fase de remisión)" (p. 86).

Se han realizado muchos estudios sobre intervención psicológica en cáncer, iniciándose en la década de los 50 y aumentando progresi- vamente a lo largo de los años. Una de las modalidades que ha ido suscitando cada vez más interés a lo largo del tiempo, por sus posibles efectos beneficiosos en la calidad de vida, ha sido la psicoterapia de grupo.

Muchos estudios han podido demostrar que la intervención psicológica en grupo puede mejorar de forma significativa el estado emocional y la calidad de vida de los pacientes oncológicos que participan en los grupos de psicoterapia. La intervención facilita el cambio de actitudes, conductas y emociones, a través de la reflexión conjunta, el intercambio de experiencias, el autoconocimiento y el apoyo mutuo (Bellver, Almonacid y Aguilar, 2004; Edmonds, Lockwood y Cunningham, 1999; Fawzy et al., 1993; Font y Rodríguez, 2007; Goodwin, 
2003; León et al., 2005; Rehse y Pukrop, 2003; Sebastián, Bueno y Mateos, 2004; Spiegel, Bloom, Kraemer y Gottheil, 1989).

Como objetivos de la psicoterapia de grupo para pacientes oncológicos se han señalado (Fawzy y Fawzy, 1998; Fobair, 1997; Gottlieb y Wachala, 2007; Johnson y Lane, 1993; Sirgo y Gil, 2000; UICC, 1998), entre otros:

1. Facilitar la expresión de emociones y pensamientos en torno a la experiencia del cáncer y sus consecuencias.

2. Facilitar la comunicación, el compartir la experiencia de la enfermedad y el apoyo mutuo entre los diferentes miembros del grupo.

3. Proporcionar apoyo emocional y social.

4. Proporcionar información sobre la enfermedad y su tratamiento.

5. Disminuir las dificultades emocionales originadas por el diagnóstico de la enfermedad y su tratamiento como ansiedad, depresión, ira, miedo, sentimientos de culpabilidad, etc.

6. Proporcionar un mayor control sobre la enfermedad.

7. Mantener o recuperar las relaciones con las personas del entorno.

8. Mejorar las relaciones de pareja.

9. Recuperar o mejorar la autoestima e imagen corporal.

10. Disminuir los sentimientos de aislamiento.

11. Facilitar estrategias de afrontamiento adecuadas.

12. Facilitar la elaboración del duelo.

13. Facilitar la adaptación a la enfermedad.

14. Mejorar la calidad de vida.
Cada vez existen más evidencias de los efectos beneficiosos de la psicoterapia de grupo como intervención psicológica dirigida a pacientes con cáncer. Entre ellos podemos destacar: la disminución de la ansiedad, de la depresión, del miedo; el aumento de las estrategias de afrontamiento activo; la mejora de la sensación de bienestar; la ayuda para resolver problemas y la mejora de la calidad de vida (Cope, 1995; Fawzy, 1999; Fobair et al., 2002; Font, 2003; Jahraus, Sokolosky, Thurston y Guo, 2002; Michalec, 2005; Rehse y Pukrop, 2003; Sebastián et al., 2004; UICC, 1998; Weis, 2003; Yoo et al., 2005).

Pero a pesar de la existencia de los grupos de psicoterapia, relativamente pocos pacientes utilizan este tipo de psicoterapia, o si participan, abandonan el tratamiento. En una revisión realizada por Gottlieb y Wachala (2007), los autores concluyen que aproximadamente el $20 \%$ de los pacientes a los que se les invita a participar en un grupo no quieren unirse a él, y otro $20 \%$ de los que participan en la psicoterapia lo dejan antes de la última sesión. Las personas que participan en estos grupos suelen ser mujeres, jóvenes, con estudios, se sienten animadas por las personas de su entorno para participar, reciben menos apoyo por parte de una persona "especial", tienen más creencias positivas sobre la participación, perciben menos dificultades para unirse a un grupo, y al mismo tiempo, perciben mayor control sobre la enfermedad y utilizan más estrategias adaptativas de afrontamiento.

Resultados similares encontraron Owen, Goldstein, Lee, Breen y Rowland (2007), estos autores hallaron que el $23,7 \%$ de los supervi- 
vientes de cáncer participaban en grupos de psicoterapia. Los predictores de la participación fueron ser mujer, con estudios, persona que utilizaba terapias alternativas, y que presentaba depresión y ansiedad. El porcentaje de participantes que refirieron que su médico les había recomendado un grupo de psicoterapia fue bajo (10,2\%).

En un estudio (Sánchez, Torres, Camprubí, Vidal y Salamero, 2005) donde se valoraron los factores predictores del abandono del grupo de psicoterapia entre mujeres con cáncer de mama, se encontró que aproximadamente un tercio de las pacientes que decidían participar, abandonaban antes de finalizar el mismo. El factor más importante que predijo el abandono fue un bajo nivel de estudios. Por otra parte, las personas con un estilo de afrontamiento activo acostumbraban a asistir a más sesiones de tratamiento grupal.

Estos resultados nos llevan a reflexionar y a intentar conocer qué procesos pueden intervenir en la psicoterapia de grupo que contribuyan a que la terapia sea eficaz, y de alguna manera, poder predecir una disminución en la tasa de abandono. Ya la investigación de Lambert (1986), identificaba en su momento que del total del cambio que experimenta el paciente en psicoterapia: (a) un $40 \%$ se debe a factores extraterapéuticos; (b) un 30\% se debe a factores comunes; (c) un 15\% es resultado de las técnicas aplicadas en la psicoterapia; y (d) otro 15\% proviene del efecto placebo (Botella, Pacheco, Herrero y Corbella, 2000). Asimismo, en el ámbito de la psicoterapia de grupo, Vinogradov y Yalom (1996) y Yalom (1985) sostienen que los factores terapéuticos de un grupo podían defi- nirse como: (a) universalidad, i.e., la conciencia de que el paciente no es único en su desdicha; (b) infundir esperanza, i.e., una elevada expectativa de ayuda antes de empezar la psicoterapia se correlaciona significativamente con un resultado positivo de la psicoterapia; (c) información participada, i.e., la importancia de la explicación de determinados fenómenos como el primer paso para el control de los mismos; y (d) altruismo, i.e., los grupos ofrecen apoyo, consuelo, complicidad entre sus participantes.

Nuestro objetivo en este estudio, es conocer más a fondo los microprocesos que contribuyen a que la psicoterapia sea eficaz. Este estudio se basa en la idea de que las teorías de los clientes sobre qué les ayuda en psicoterapia también son relevantes para su curso (Brykczysnka, 1990; Mohr y Woodhouse, 2000; Watson y Rennie, 1994; entre otros). Tradicionalmente, este tipo de estudios de procesos han estado centrados en la visión de los terapeutas sobre el cambio (véase Kleinke, 1994, para una revisión de dichos estudios). Nuestro interés, en cambio, se centra en las teorías de las clientes sobre el cambio y, más concretamente, sobre los acontecimientos de ayuda o de no ayuda en nuestros propios grupos.

Así pues, nuestro objetivo es analizar y describir: (a) aspectos significativos o de ayuda y (b) aspectos no significativos o de no ayuda en grupos de psicoterapia realizados con mujeres con cáncer de mama, enfocados desde una epistemología constructivista relacional (BoteIla, 2000; Botella y Herrero, 2000). 
Método

\section{Sujetos y material}

La muestra está compuesta por 50 mujeres con cáncer de mama (edad media: 45 años; DS $=8^{\prime} 7$ ) que participaron en uno de los cuatro grupos de psicoterapia analizados y que describimos a continuación.

Los grupos eran conducidos por un equipo de terapeutas formado por un terapeuta y un coterapeuta que dirigían los grupos y un grupo de psicoterapeutas que los supervisaban extrasesión (todos los miembros del equipo tenían una formación en terapia cognitivoconstructivista, narrativa y sistémica). Una de las psicoterapeutas fue la misma en todos los grupos mientras que el/la coterapeuta fue una persona diferente en cada grupo. El trabajo de grupo se realizaba en 10 sesiones, una vez a la semana, durante 2 horas con un promedio de 10 pacientes por grupo.

Los grupos eran cerrados aunque se permitieron nuevas incorporaciones cuando se produjeron en una segunda o tercera sesión, si se consideraba oportuno según el equipo de psicoterapeutas y previo consentimiento del propio grupo de pacientes.

Los grupos se inspiraron en los grupos de transacción interpersonal (IT Groups) introducidos inicialmente por Landfield y Rivers (1975) como grupos de apoyo psicoterapéutico para estudiar y mejorar las relaciones sociales. En este sentido, se trataba de una actividad basada en sesiones grupales alrededor de temas de interés general relacionados con la vida cotidiana en general y con las relaciones interpersonales en particular.
El objetivo principal de este grupo de psicoterapia consistía en ayudar a las participantes a mejorar su calidad de vida y afrontar las situaciones estresantes y conflictivas de forma más constructiva, atribuyendo nuevos y más adaptativos significados a sus vidas y a las implicaciones del diagnóstico de cáncer en las mismas. Asimismo, un segundo objetivo básico de este grupo era el de fomentar las relaciones interpersonales entre las participantes de forma que la propia actividad constituyera una experiencia social enriquecedora de apoyo y cohesión y reconstrucción de significados.

La estructura y contenidos de nuestros grupos así como la experiencia clínica con los mismos, coincide con el trabajo desarrollado por Spira y Reed (2003) sobre los motivos de demanda más frecuentes entre mujeres con cáncer de mama y el impacto del diagnóstico de cáncer en sus vidas. A continuación se describe brevemente ambas categorías según dichos autores: (a) algunos de los motivos de demanda más comunes en los grupos de mujeres con cáncer son: "me siento sola", "me gustaría comunicarme mejor con la gente", "me gustaría ser capaz de expresar mis sentimientos; hasta ahora me lo guardaba todo dentro, me lo tragaba todo","me gustaría ser capaz de afirmarme a mí misma, de decir 'no' y de no sentirme dominada por los demás: quiero aprender a hacerme valer", "me gustaría ser capaz de acercarme emocionalmente a los demás", "me gustaría ser capaz de confiar en los demás, me han hecho sufrir bastante a menudo en la vida", "me gustaría saber qué impresión causo a los demás"; y (b) El trabajo con el impacto del diagnóstico de cáncer y sus consecuencias en: la 
vida de la cliente y su relación consigo misma (en general), la relación con su imagen, la relación con su tratamiento y el personal sanitario (especialmente con su médico), la relación con su familia y otras personas significativas, la relación con su pareja, la relación con su sexualidad, la relación con su vida laboral y/o tareas del hogar, la relación con su futuro, la relación con la muerte y la finitud y en la relación con el grupo de psicoterapia y su final.

Con el fin de alcanzar estos objetivos el grupo se definió por los siguientes subobjetivos:

- Ayudar a las participantes a comprender aquellos comportamientos que pueden derivar en problemas en las relaciones con los demás.

- Plantear formas nuevas de afrontar los conflictos o situaciones difíciles.

- Compartir aquello que las angustia.

El grupo pretendía, pues, de manera global, ofrecer formas diferentes y significativas de afrontar las situaciones de la vida cotidiana en un contexto grupal que fomentaba el enriquecimiento de todas sus participantes y que se basaba en el diálogo.

Teniendo en cuenta que los contenidos se trabajaron en grupo eran previamente negociados entre participantes y terapeutas, se exponen a continuación algunos de los temas que resultaron de más interés general entendidas sus implicaciones para la vida cotidiana y las relaciones con los demás: vinculación, asertividad, dolor, confianza, control, poder, identidad, personas que me influyen, aprobación, autoestima, imagen corporal, rabia, ansiedad y estrés, miedo, conflicto y relaciones de intimidad y pareja.

\section{Procedimiento}

Tras algunas de las sesiones de grupo, se solicitaba a las participantes escribir sobre los acontecimientos de ayuda y de no ayuda al finalizar la sesión.

El análisis cualitatitivo se realizó mediante el método de grounded theory (Corbin, 1998; Glaser y Strauss, 1967; Madill, Jordan y Shirley, 2000; Rennie, 1998a, 1998b, 1999, 2000, 2001).

En primer lugar, se dividió las unidades de análisis dependiendo de a lo que se referían. Como resultado de estos cuatro grupos de psicoterapia, se recogieron 319 unidades de análisis (319 sentencias). De todas ellas, 241 se referían a acontecimientos de ayuda mientras que 78 hacían referencia a acontecimientos no significativos. Los acontecimientos significativos y no significativos se consideran categorías centrales. Las demás categorías que desarrollamos dependían de estas dos categorías de estructura jerárquica superior.

En segundo lugar, una vez las unidades se codificaban en estos dos grandes grupos, los analistas codificaban cada unidad en términos de su significado, intentando ser lo más precisos posibles al contenido de la unidad. Ello se realizó resumiendo primero el significado de cada unidad.

En tercer lugar, los analistas procedieron a comparar los significados de estas unidades con el objetivo de encontrar los aspectos comunes entre ellos. Una vez localizados, se les dió una etiqueta y cada una de ellas se confirmó en términos de estas categorías. Como recomendaron Rennie, Phillips y Quartaro (1988), en los primeros pasos del análisis se describieron estas categorías y los analistas utilizaron el mismo lenguaje de las participantes. 
En cuarto lugar, los analistas realizaron un segundo resumen de estas categorías utilizando términos que les ayudaron a explicar las categorías descriptivas y las relaciones entre ellas (Collaizi, 1979; Sullivan, 1984). Cuando aparecía una nueva categoría, cada unidad de análisis se comparaba a ellas y se codificaban de nuevo en una "categorización abierta" (Rennie et al., 1988, p. 143).

Finalmente, todos estos análisis se conformaban en una estructura de mapa jerárquico donde las categorías inferiores eran consideradas como propiedades de las superiores (las últimas incluyen las categorías formadas). Cuando los datos ya no proporcionan información adicional al análisis, la taxonomía se considera como saturada y finaliza el análisis.

Se debería mencionar que cuatro terapeutas diferentes realizaron el análisis cualitativo con una actitud genuina con el objetivo de asegurar la fiabilidad del estudio. Las unidades de los análisis sólo se codificaban cuando existía un acuerdo entre todos los terapeutas.

\section{Resultados}

Los resultados se exponen en la Figura 1 y en la 2. La 1 corresponde a los acontecimientos de ayuda, mientras la 2 al mapa del análisis de los acontecimientos de no ayuda. Entre paréntesis aparecen la cantidad de unidades de análisis codificadas en cada categoría. La suma

Mapa de acontecimientos de ayuda. Los acontecimientos significativos en el grupo se atribuyen a:

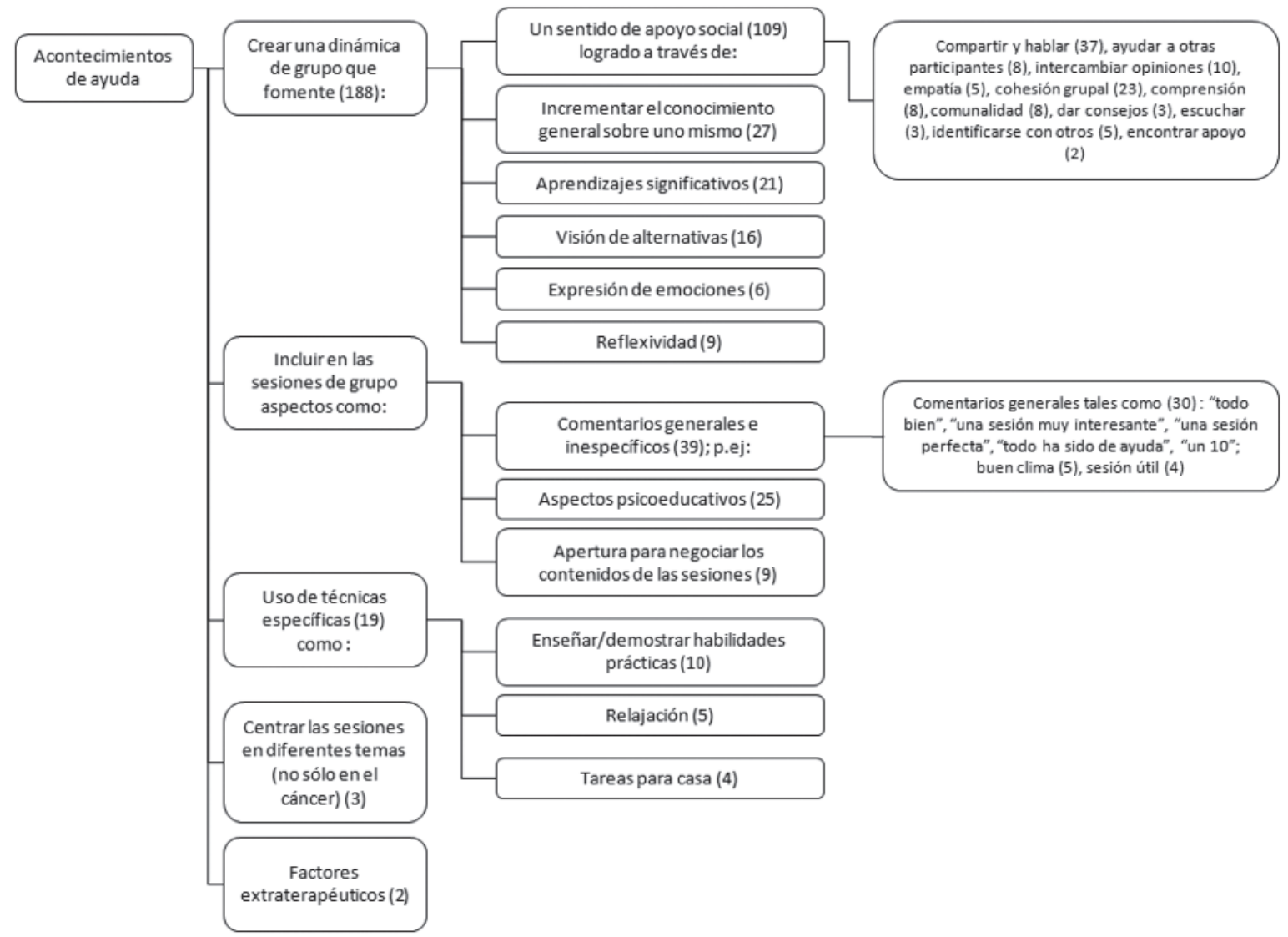

Figura 1. Acontecimientos de ayuda. 
Mapa de acontecimientos que no ayudan. Los acontecimientos no significativos se atribuyen a:

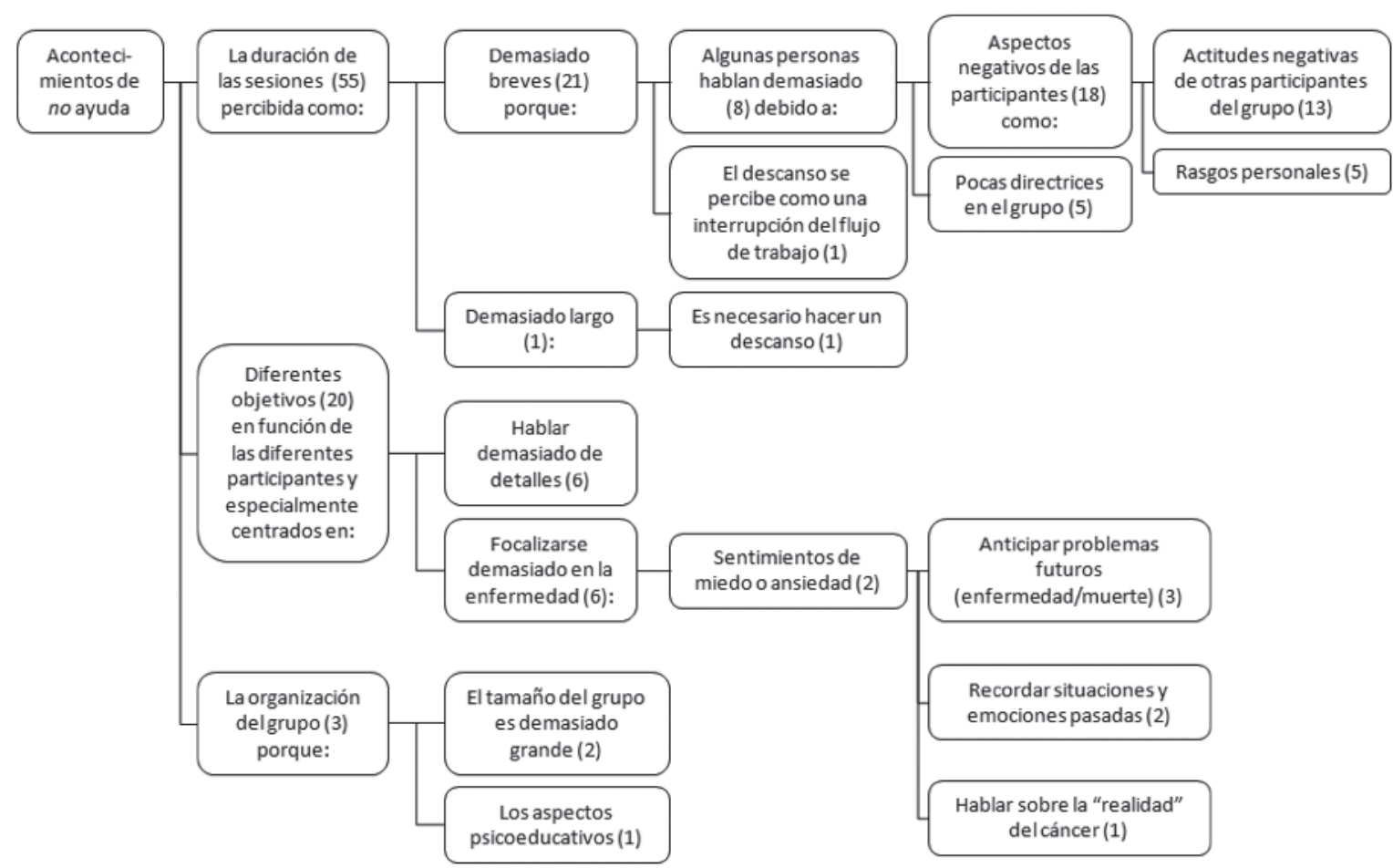

Figura 2. Acontecimientos de no ayuda.

de los números entre paréntesis no corresponde exactamente con el número que aparece en las categorías supranucleares, puesto que cada unidad de análisis puede ser codificada en más de una categoría, y las cantidades de unidades codificadas en las categorías supra pueden ser independientes de las codificadas en categorías infra.

\section{Discusión}

Coherentemente con la perspectiva constructivista relacional (Botella, 2000; Botella y Herrero, 2000) desde la que se ha enfocado el trabajo psicoterapéutico en los grupos, también la discusión se realizará desde el mismo marco epistemológico.

Como se puede observar en los dos mapas, las participantes identifican como elemento terapéutico al propio grupo (como un todo), y

no a la suma de las personas que lo forman. Los aspectos positivos en estos grupos psicoterapéuticos no tienen que ver con la identificación de características positivas de las pacientes que participan en ellos, sino con el grupo en sí mismo (incluyendo tanto a clientes como a psicoterapeutas). Ello nos lleva al concepto de responsabilidad relacional (Gergen, 1999), que el grupo funcione dependerá de la relación en sí misma y no de la suma de las contribuciones aisladas de las personas que lo forman.

Cuando se refiere a los acontecimientos de ayuda existe la sensación de que las personas se ven a sí mismas como "constituyentes de un proceso que eclipsa a cualquier individuo del mismo pero que es, a su vez, constituido por sus elementos individuales" (Gergen, 1999, p. 129). Existe un sentimiento de "autodistribución", es decir, el grupo es 
visto como una comunidad que piensa como un solo sujeto.

También encontramos una sensación de acción coordinada (Gergen, 1999), es decir, el grupo siente que se mueve en armonía para generar significados juntos a través de "compartir y hablar", "cohesión grupal", "comunalidad" o "empatía", tal y como puede observarse en la primera línea del mapa de los resultados que mostramos en la Figura 1. De este modo, podemos afirmar que tiene lugar un diálogo transformador (Gergen, 1999) en el que las participantes sienten que se reconocen en las vivencias de las demás, en un proceso de co-constituirse y moverse hacia un sentido de mutualidad. Esto lleva a las participantes a sentirse más próximas a las demás, a sentirse como "una" -tal y como se ha mencionado anteriormente- a través de conversaciones donde puedan emerger nuevas posiciones subjetivas. La psicoterapia tiene lugar en el lenguaje $y$, en este sentido, se puede equiparar a un diálogo colaborativo destinado a transformar las narrativas de identidad de los clientes. A pesar de la popularidad del modelo médico y de las metáforas que inspira, la psicoterapia no deriva su potencial transformador de ser un tratamiento o una cura, sino de ser una forma especializada de conversación en que -como se afirmaba- "se da voz a nuevas posiciones subjetivas, se narran nuevas historias, emergen nuevas formas de inteligibilidad y lo aún no dicho, encuentra un lugar para ser oído consciente y reflexivamente" (Botella, 2000; Botella y Herrero, 2000, p. 9).

La habilidad del terapeuta es la de liberar espacios para que tenga lugar este diálogo transformador. No hace falta que sea un técnico hábil o estratégico; sus metas no han de separarle del cliente convirtiéndole en alguien cuyo único interés reside en modificar sus conductas, hacerlas más racionales, reestructurar su sistema familiar o iluminarlo sobre los intrincados detalles de sus procesos inconscientes. Si bien todas las anteriores pueden ser metas valiosas e interesantes en un momento dado de una relación terapéutica en desarrollo, también pueden convertirse con demasiada facilidad en narrativas totalizantes si el terapeuta cree en ellas con excesiva fe. En el momento en que el terapeuta se las tome demasiado en serio y olvide que son juegos de lenguaje cuyo poder deriva de su capacidad para llevar la conversación más allá, pueden llegar a ahogar todo lo que hay de nuevo y fresco en el diálogo terapéutico (Botella, 2000; Botella y Herrero, 2000).

Desde el punto de vista del constructivismo relacional, el terapeuta ha de ser alguien capaz de utilizar la palabra para dar vida a nuevos juegos de lenguaje, juegos que permitan al cliente recuperar el sentimiento perdido de inteligibilidad y transformación. En este sentido, como anticipó George Kelly en los años sesenta: (...) la psicoterapia es una forma de embarcarse en la aventura humana y puede encarnar y movilizar todas las técnicas útiles para tal aventura que se hayan diseñado. Desde luego no existe una única técnica psicoterapéutica ni una única forma de compatibilidad personal entre terapeuta y cliente. Las técnicas empleadas deben ser las técnicas que usamos para vivir, y la tarea del terapeuta hábil es la orquestación adecuada de todas ellas (Kelly, 1969, p. 222). 
El grupo de psicoterapia es, así, visto como un proceso de construcción conjunta de significado, es decir, una forma de juego al que jugamos juntos (Wittgenstein, 1953) y, por esta razón, como un producto relacional de prácticas discursivas compartidas y acciones conjuntas (Botella, 2000; Botella y Herrero, 2000). A través de un proceso de suplementación (Gergen, 1994) las personas aprenden más sobre ellas mismas, sus formas de comportarse... tal y como indica el mapa de acontecimientos de ayuda con las categorías "aumentar el conocimiento general sobre uno mismo" o "aprendizaje significativo". Los "aspectos psicoeducativos" y "utilizar técnicas específicas" también son percibidos de forma significativa cuando se refieren a aspectos de ayuda. A través de los aspectos psicoeducativos y sus técnicas, las personas conciben que están aprendiendo más de sí mismas así como realizando aprendizajes significativos que serán útiles en sus vidas.

La psicoterapia se puede ver como una liberación de la inmersión en construcciones que limitan y constriñen el mundo (Gergen y Kaye, 1996), debido a la apertura de las participantes a la receptividad de lo que las demás tienen que decir en el grupo. En los grupos psicoterapéuticos, las participantes ven las múltiples perspectivas presentadas por el diálogo con las demás como un acontecimiento de ayuda, como se puede observar en las categorías utilizadas en el mapa como: "visión de alternativas", "intercambio de opiniones" o "dar consejos". Sin embargo, e interesantemente, pocas personas consideran la "reflexividad" como un aspecto de ayuda.
En contraste con los resultados de los aspectos positivos, todos los aspectos negativos hacen referencia a la vivencia del grupo como formado por sujetos individuales. Así, los aspectos que se perciben como disfuncionales para el grupo se traducen en rasgos o actitudes de una persona u otra del grupo como se puede ver en la categoría "Aspectos negativos de las participantes" y "obviamente, culparse mútuamente es un impedimento para la responsabilidad relacional" (Gergen, 1999, p. 157).

Esto también se puede observar en la categoría "Diferentes objetivos en función de las diferentes participantes". Cuando se refiere a acontecimientos de no ayuda, el grupo no se mueve hacia un objetivo construido conjuntamente sino hacia diferentes aspectos en términos de los distintos intereses individuales. Aparece la sensación de personas independientes que se encuentran, justo lo contrario que sucede cuando analizamos los aspectos de ayuda. Emergen posiciones antagónicas, las participantes se posicionan a sí mismas como las personas que saben cómo debería funcionar el grupo, y posicionan a las demás como personas que no se preocupan por el buen clima del grupo.

Finalmente, algunas unidades del análisis contienen sugerencias sobre el aumento del número de sesiones. Esto tiene que ver con el hecho de que el grupo haya conseguido una cohesión significativa y de que se han compartido emociones intensas y experiencias relevantes durante las diez sesiones. Así, el grupo solicita más sesiones con el objetivo de continuar trabajando en los cambios deseados, el 
grupo siente el final de la psicoterapia como prematuro considerando los cambios que se han ido realizando. Además, el grupo es considerado por sus participantes como el espacio idóneo en el que poder hablar sobre las propias necesidades y en el que poder sentir la comprensión de personas que se encuentran en situaciones similares.

Llegados a este punto, podemos concluir que el estudio de los aspectos de ayuda y de no ayuda expresados por las pacientes coinciden con la visión tradicional de los psicoterapeutas sobre lo que sirve o no de ayuda dentro de un grupo de psicoterapia; de este modo, los resultados de este estudio contribuyen a confirmar la validez de las teorías sobre los factores de ayuda en los grupos de psicoterapia con la aportación de la visión de los propios pacientes.

\section{Bibliografía}

Andersen, B. L. (1992). Psychological interventions for cancer patients to enhance the quality of life. Journal of Consulting and Clinical Psychology, 60, 552-568.

Avis, N. E., Crawford, S., \& Manuel, J. (2004). Psychosocial problems among younger women with breast cancer. Psycho-Oncology, 13, 295-308.

Baider, L. (1995). Psychological intervention with couples after mastectomy. Supportive Care in Cancer, 3, 239-243.

Bárez, M. (2002). Relación entre percepción de control y adaptación a la enfermedad en pacientes con cáncer de mama. Tesis doctoral no publicada. Universitat Autònoma de Barcelona. Bellaterra: Barcelona.

Bellver, A. (2007). Eficacia de la terapia grupal en la calidad de vida y el estado emocional en mujeres con cáncer de mama. Psicooncología, 4, 133-142.
Bellver, A., Almonacid, V., \& Aguilar, P. (2004, agosto). Group psychotherapy for women with breast cancer. Trabajo presentado en el 7th World Congress of Psycho-Oncology, Dinamarca.

Botella, L. (2000). Diálogo, relaçoes e mudança: Una aproximaçao discursiva à psicoterapia constructivista. En Ó. Gonçalves \& M. Gonçalves (Eds.), Abordagens construcionistas à psicoterapia. Coimbra: Quarteto.

Botella, L., \& Herrero, O. (2000). A relational constructivist approach to narrative therapy. European Journal of Psychotherapy, Counselling and Health, 3, 407-418.

Botella, L., Pacheco, M., Herrero, O. \& Corbella, S. (2000). Investigación en psicoterapia: El estado de la cuestión. Boletín de Psicología, 69, 19-42.

Bower, J. E., Ganz, P. A., Desmond, K. A., Bernaards, C. A., Rowland, J. H., Meyerowitz, B. $E$. et al. (2006). Fatigue in long-term breast carcinoma survivors. A longitudinal investigation. Cancer, 106, 751-758.

Boyle, P. \& Ferlay, J. (2004). Cancer incidence and mortality in Europe. Annals of Oncology, 16, 481-488.

Brykczynska, C. (1990). Changes in the patient's perception of his therapist in the process of group and individual psychotherapy. Psychotherapy and Psychosomatics, 53, 179184.

Bultz, B. D., Speca, M., Brasher, P. M., Geggie, P. H. S., \& Page, S. A. (2000). A randomized controlled trial of a brief psychoeducational support group for partners of early stage breast cancer patients. Psycho-Oncology, 9, 303-313.

Cabanes, A., Pérez-Gómez, B., Aragonés, N., Pollán, M. \& López-Abente, G. (2009). La situación del cáncer en España. 1975-2006. Madrid: Centro Nacional de Epidemiología. Instituto de Salud Carlos III.

Cameron, L. D., Booth, R. J., Schlatter, M., Ziginskas, D., \& Harman, J. E. (2007). Changes in emotion regulation and psychological ad- 
justment following use of a group psychosocial support program for women recently diagnosed with breast cancer. Psycho-Oncology, 16, 171-180.

Carlsson, M., \& Hamrin, E. (1994). Psychological and psychosocial aspects of breast cancer and breast cancer treatment. A literature review. Cancer Nursing, 17, 418-428.

Cimprich, B., Janz, N. K., Northouse, L., Wren, P. A., Given, B., \& Given, C.W. (2005). Taking Charge: A self-management program for women following breast cancer treatment. Psycho-Oncology, 14, 704-717.

Collaizi, P. F. (1979). Psychological research as the phenomenologist views it. En R. S. Valle \& M. King (Eds.), Existential phenomenological alternatives for psychology (pp. 48-71). New York: Oxford University Press.

Cope, D. G. (1995). Functions of a breast cancer support group as perceived by the participants: An ethnographic study. Cancer Nursing, 18, 472-478.

Corbin, J. (1998). Alternative interpretations:Valid or not?. Theory and Psychology, 8, 121-128.

Cruzado, J. A. \& Olivares, Ma. E. (1996). Intervención psicológica en pacientes con cáncer. En J. M. Buceta \& A. M. Bueno (Eds.), Tratamiento psicológico de hábitos y enfermedades (pp. 495-543). Madrid: Pirámide.

Curado, M. P., Edwards, B., Storm, H. \& Boyle, P. (2007). Cancer Incidence in five continents. IARC,CancerBase, No 160, Lyon.

Edmonds, C. V. I., Lockwood, G. A., \& Cunningham, A. J. (1999). Psychological response to long term group therapy: A randomized trial with metastatic breast cancer patients. Psycho-Oncology, 8, 74-91.

Fawzy, F. I. (1999). Psychosocial interventions for patients with cancer: What works and what doesn't. European Journal of Cancer, 35, 1559-1564.

Fawzy, F. I. \& Fawzy, N. W. (1998). Group therapy in the cancer setting. Journal of Psychosomatic Research, 45, 191-200.
Fawzy, F. I., Fawzy, N. W., Arndt, L. A., \& Pasnau, R. O. (1995). Critical review of psychosocial interventions in cancer care. Archives of General Psychiatry, 52, 100-113.

Fawzy, F. I., Fawzy, N. W., Hyun, C. S., Elashoff, R., Guthrie, D., Fahey, J. L. et al. (1993). Malignant melanoma. Effects of an early structured psychiatric intervention, coping and affective state on recurrence and survival 6 years later. Archives of General Psychiatry, 50, 681-689.

Ferlay, J., Bray, F., Pisani, P., \& Parkin, D. M. (2004). GLOBOCAN 2002: Cancer Incidence, Mortality and Prevalence Worldwide. IARC CancerBase No. 5. version 2.0, IARCPress, Lyon.

Ferrell, B. R., Grant, M. M., Funk, B. M., OtisGreen, S. A., \& García, N. J. (1998). Quality of life in breast cancer survivors: Implications for developing support services. Oncology Nursing Forum, 25, 887-895.

Fobair, P. (1997). Cancer support groups and group therapies: Part II. Process, organizational, leadership and patient issues. Journal of Psychosocial Oncology, 15, 123-147.

Fobair, P., Koopman, C., Dimiceli, S., O'Hanlan, K., Butler, L., Classen, C. et al. (2002). Psychosocial intervention for lesbians with primary breast cancer. Psycho-Oncology, 11, 427-438.

Font, A. (2003). Guía de tratamientos psicológicos eficaces en el cáncer. En M. Pérez, J. R., Fernández, C., Fernández \& I. Amigo (Coords.), Guía de tratamientos psicológicos eficaces II. Psicología de la salud (pp. 57-92). Madrid: Pirámide.

Font, A. \& Rodríguez, E. (2007). Eficacia de las intervenciones psicológicas en mujeres con cáncer de mama. Psicooncología, 4, 423-446.

Fundación Científica de la Asociación Española contra el Cáncer (2005). Globocan 2000. El cáncer en el mundo. Recuperado el 30 de agosto del 2005 en http://wwwdep.iarc.fr/globocan/table2.asp?cancer= $132 \&$ region $=99 \&$ sex $=2 \&$ sort . 
García-Lumbreras, S. \& Blasco, T. (2008). Características de la sexualidad en mujeres con cáncer de mama. Psicooncología, 5, 155170.

Gergen, K. J. (1994). Realities and relationship. Cambridge, MA: Harvard University Press.

Gergen, K. J. \& Kaye, J. (1996). Más allá de la narración en la negociación del significado terapéutico. En S. MacNamee y K. J. Gergen (Comps.), La terapia como construcción social (pp. 119-218). Barcelona: Paidós.

Gergen, K. J. (1999). An invitation to social construction. Thousand Oaks: Sage.

Gielissen, M. F. M., Verhagen, S., Witjes, F., \& Bleijenberg, G. (2006). Effects of cognitive behavior therapy in severely fatigued disease-free cancer patients compared with patients waiting for cognitive behavior therapy: A randomized controlled trial. Journal of Clinical Oncology, 24, 4882-4887.

Given, C., Given, B., Rahbar, M., Jeon, S., McCorkle, R., Cimprich, B. et al. (2004). Effect of a cognitive behavioural intervention on reducing symptom severity during chemotherapy. Journal of Clinical Oncology, 22, 507-516.

Glaser, B. G. \& Strauss, A. (1967). The discovery of grounded theory: Strategies for qualitative research. Chicago: Aldine.

Goodwin, P. J. (2003). Psychosocial support for women with advanced breast cancer. Breast Cancer Research and Treatment, 81 (Suppl. 1), 103-110.

Gottlieb, B. H. \& Wachala, E. D. (2007). Cancer support groups: A critical review of empirical studies. Psycho-Oncology, 16 (5), 379400.

Greenstein, M. \& Breitbart, W. (2000). Cancer and the experience of meaning: A group psychotherapy program for people with cancer. American Journal of Psychotherapy, 54, 486-500.

Helgeson, V. S. \& Tomich, P. L. (2005). Surviving cancer: A comparison of 5 year disease-free breast cancer survivors with healthy women. Psycho-Oncology, 14, 307-317.

Hodgkinson, K., Butow, P., Hunt, G. E., Pendlebury, S., Hobbs, K. M., \& Wain, G. (2007). Breast cancer survivors' supportive care needs 2-10 years after diagnosis. Supportive Care in Cancer, 15, 515-523.

Hogan, B. E., Linden, W., \& Najarian, B. (2002). Social support interventions. Do they work? Clinical Psychology Review, 22, 381-440.

Institut Català d'Oncologia (2001a). Càncer Catalunya 2001. Generalitat de Catalunya. Departament de Sanitat i Seguretat Social.

Institut Català d'Oncologia (2001 b). Plà Director d'Oncologia a Catalunya: 2001-2004. Servei Català de la Salut.

Izquierdo, A., Gispert, R., Saladie, F. \& Espinàs, J.A. (2008). Análisis de la incidencia, la supervivencia y la mortalidad según las principales localizaciones tumorales, 19852019: cáncer de mama. Medicina Clínica (Barcelona), 131 (supl. 1), 50-52.

Jahraus, D., Sokolosky, S., Thurston, N., \& Guo, D. (2002). Evaluation of an education program for patients with breast cancer receiving radiation therapy. Cancer Nursing, 25, 266-275.

Johnson, J. \& Lane, C. (1993). Role of support groups in cancer care. Supportive Care in Cancer, 1, 52-56.

Kelly, G. A. (1969). The psychotherapeutic relationship. En B. A. Maher (Ed.), Clinical psychology and personality: The selected papers of George Kelly (pp. 216-223). New York: Krieger.

Kissane, D. W., Bloch, S., Smith, G. C., Miach, P., Clarke, D. M., Ikin, J. et al. (2003). Cognitiveexistential group psychotherapy for women with primary breast cancer: A randomised controlled trial. Psycho-Oncology, 12, 532-546.

Kleinke, C. L. (1994). Common principles of psychotherapy. Belmont CA: Wadsworth.

Kuijer, R. G., Buunk, B. P., De Jong, G. M., Ybema, J.F., \& Sanderman, R. (2004). Effects of 
a brief intervention program for patients with cancer and their partners on feelings of inequity, relationship quality and psychological distress. Psycho-Oncology, 13, 321-334.

Lambert, M. J. (1986). Implications on psychotherapy outcome research for eclectic psychotherapy. En J. C. Norcross (Ed.), Handbook of Eclectic Psychotherapy. New York: Brunner-Mazel.

Landfield, A., \& Rivers, P.C. (1975). Interpersonal transaction and rotating dyads. Psychotherapy: Theory, Research and Practice, 12, 466437.

Larson, M. R., Duberstein, P. R., Talbot, N. L., Caldwell, C., \& Moynihan, J. A. (2000). A presurgical psychosocial intervention for breast cancer patients: Psychological distress and the immune response. Journal of Psychosomatic Research, 48, 187-194.

Lemieux, J., Bordeleau, L., \& Goodwin, P. (2007). Medical, psychosocial, and health-related quality of life issues in breast cancer survivors. En P. Ganz (Ed.), Cancer survivorship. Today and tomorrow (pp. 122-144). New York: Springer.

León, C., Domènech, J., Casimiro, N., Montiel, R., García, A., Campos, R. et al. (2005). Grupo psicoeducativo de pacientes con cáncer de mama. Ponencia presentada en el II Congreso Nacional: Psicología y Cáncer en el siglo XXI. SEPO, noviembre, Murcia.

Lindemalm, C., Strang, P., \& Lekander, M. (2005). Support group for cancer patients. Does it improve their physical and psychological wellbeing? A pilot study. Supportive Care in Cancer, 13, 652-657.

Madill, A., Jordan, A., \& Shirley, C. (2000). Objectivity and reliability in qualitative análisis: Realist, contextualist and radical constructionist epistemologies. British Journal of Psychology, 91, 1-20.

McArdle, J. M. C., George, W. D., McArdle, C. S., Smith, D. C., Moodie, A. R., Hughson, A. V. M. et al. (1996). Psychological support for pa- tients undergoing breast cancer surgery: A randomised study. British Medical Journal, 312, 813-816.

Michael, Y., Berkman, L., Colditz, G., Holmes, M., \& Kawachi, I. (2002). Social networks and health-related quality of life in breast cancer survivors. A prospective study. Journal of Psychosomatic Research, 52, 285-293.

Michalec, B. (2005). Exploring the multidimensional benefits of breast cancer support groups. Journal of Psychosocial Oncology, 23, 159-179.

Mohr, J. J., \& Woodhouse, S.S. (2000). Clients' visions of helpful and harmful psychotherapy: An approach to measuring individual differences in therapy priorities. Comunicación presentada en el $31^{\text {st }}$ Annual Meeting of the Society for Psychotherapy Research (SPR): Return to beginning, Chicago, USA.

Molassiotis, A., Yung, H. P., Yam, B. M. C., Chan, F. Y. S., \& Mok, T. S. K. (2002). The efectiveness of progressive muscle relaxation training in managing chemotherapy-induced nausea and vomiting in Chinese breast cancer patients: A randomised controlled trial. Supportive Care in Cancer, 10, 237-246.

Mols, F., Vingerhoets, A. J. J. M., Coebergh, J. W., \& Van de Poll-Franse, L. V. (2005). Quality of life among long-term breast cancer survivors: A systematic review. European Journal of Cancer, 41, 2613-2619.

Moorey, S., Greer, S., Bliss, J., \& Law, M. (1998). A comparison of adjuvant psychological therapy and supportive counselling in patients with cancer. Psycho-Oncology, 7, 218-228.

Moorey, S., Greer, S., Watson, M., Baruch, J. D. R., Robertson, B. M., Mason, A. et al. (1994). Adjuvant psychological therapy for patients with cancer: Outcome at one year. PsychoOncology, 3, 39-46.

Neyt, M., \& Albrecht, J. (2006). The long-term evolution of quality of life for disease-free breast cancer survivors: A comparative study in Belgium. Journal of Psychosocial Oncology, 24 (3), 89-123. 
Okamura, H., Fukui, S., Nagasaka, Y., Koike, M., \& Uchitomi, Y. (2003). Psychoeducational intervention for patients with primary breast cancer and patient satisfaction with information: An exploratory analysis. Breast Cancer Research and Treatment, 80, 331-338.

Owen, J. E., Goldstein, M. S. Lee, J. H., Breen, N., \& Rowland, J. H. (2007). Use of healthrelated and cancer-specific support groups among adult cancer survivors. Cancer, 109, 2580-2589.

Parkin, D. M., Whelan, S., Ferlay, J., \& Storm, H. (2005). Cancer incidence in five continents. Vol. I to VIII. IARC CancerBase, 7, Lyon.

Pugliese, P., Fabi, A., Maggi, G., Falcicchio, C., \& Cognetti, F. (2006, octubre). Impact of surgery and adjuvant treatment on the quality of life of women with breast carcinoma: $A$ prospective study. Trabajo presentado en el 8th World Congress of Psycho-Oncology, Venecia.

Rehse, B., \& Pukrop, R. (2003). Effects of psychosocial interventions on quality of life in adult cancer patients: Meta analysis of 37 published controlled outcome studies. Patient Education and Counseling, 50, 179-186.

Rennie, D. L. (1998a). Grounded theory methodology: The pressing need for a coherent logic of justification. Theory and Psychology, 8, 101-119.

Rennie, D. L. (1998b). Person-centered counseling: An experiential approach. Thousand Oaks, CA: Sage.

Rennie, D. L. (1999). Qualitative research: A matter of hermeneutics and the sociology of knowledge. En M. Kopala \& L. Suzuki (Eds.), Using qualitative methods in psychology (pp. 3-13). Thousand Oaks, CA: Sage.

Rennie, D. L. (2000). Grounded theory methodology as methodical hermeneutics: Reconciling realism and relativism. Theory and Psychology, 10, 481-502.

Rennie, D. L. (2001). Grounded theory methodology as methodical hermeneutics: Reconciling realism and relativism. En J.
Frommer \& D. L. Rennie (Eds.), Qualitative psychotherapy research: Methods and methodology (pp. 32 a 49). Lengerich: Pabst Science Publishers.

Rennie, D. L., Phillips, J. R., \& Quartaro, G. K. (1988). Grounded theory: A promising approach to conceptualization in psychology? Canadian Psychology, 29, 139-150.

Roffe, L., Schmidt, K., \& Ernst, E. (2005). A systematic review of guided imagery as an adjuvant cancer therapy. Psycho-Oncology, 14, 607-617.

Rosedale, M., \& Krainovich-Miller, B. (2008). Confronting the unexpected more than a year following breast cancer treatment: $A$ secondary analysis of phenomenological data. Ponencia presentada en el 10th World Congress of Psycho-Oncology, junio, Madrid.

Salvatierra, V. (1995). Repercusiones psicológicas del cáncer de mama. Cuadernos de Medicina Psicosomática, 33, 6-21.

Sánchez, N., Torres, A., Camprubí, N., Vidal, A. \& Salamero, M. (2005). Factores predictores del abandono de la intervención psicológica grupal en una muestra de pacientes con cáncer de mama. Psicooncología, 2, 21-32.

Sant, M., Allemani, C., Santaquilani, M., Knijn, A., Marchesi, F., \& Capocaccia, R. (2009). EUROCARE-4. Survival of cancer patients diagnosed in 1995-1999. Results and commentary. European Journal of Cancer, 45, 931-991.

Schnoll, R. A., Knowles, J. C., \& Harlow, L. (2002). Correlates of adjustment among cancer survivors. Journal of Psychosocial Oncology, 20, 37-59.

Sebastián, J., Bueno, Ma. J. \& Mateos, N. (2004). Apoyo emocional y calidad de vida en mujeres con cáncer de mama. Factores psicosociales en el cáncer de mama. Madrid: Programa de Salud y Servicios Sociales, Subdirección general de programas, Instituto de la Mujer.

Sebastián, J., Bueno, Ma.J., Mateos, N. \& García, P. (1999). Evaluación de un programa de intervención sobre cáncer de mama. Boletín de Psicología, 65, 53-73. 
Sirgo, A. \& Gil, F. (2000). Intervención psicoeducativa y terapia de grupo en pacientes con cáncer. En F. Gil (Ed.), Manual de PsicoOncología (pp. 57-74). Madrid: Nova Sidonia Oncología.

Spiegel, D., Bloom, J. R., Kraemer, H. C., \& Gottheil, E. (1989). Effect of psychosocial treatment on survival of patients with metastasic breast cancer. The Lancet, 888-891.

Spira, J. L., \& Reed, G. M. (2003). Group psychotherapy for women with breast cancer. Washington DC: American Psychological Association.

Sullivan, E. V. (1984). A critical psychology. New York: Plenum Press.

Stanton, A. L. (2006). Psychosocial concerns and interventions for cancer survivors. Journal of Clinical Oncology, 24, 5132-5137.

Tartaro, J., Roberts, J., Nosarti, C., Crayford, T., Luecken, L., \& David, A. (2005). Who benefits? Distress, adjustment and benefit-finding among breast cancer survivors. Journal of Psychosocial Oncology, 23, 45-64.

UICC (1998). Workhop 1. Patient support groups. En Psycho-social impacts of breast cancer. Suiza: Autor.

Vinogradov, S. \& Yalom, I.D. (1996). Guía breve de psicoterapia de grupo. Barcelona: Paidós.

Vivar, C. G., \& McQueen, A. (2005). Informational and emotional needs of long-term survivors of breast cancer. Journal of Advanced Nursing, 51, 520-528.

Watson, J. C., \& Rennie, D. L. (1994). Qualitative analysis of client's subjective experience of significant moments during the exploration of problematic reactions. Journal of Counseling Psychology, 41, 500-509.

Weis, J. (2003). Support groups for cancer patients. Supportive Care in Cancer, 11, 763768.

Wilson, R. W., Taliaferro, L. A., \& Jacobsen, P. B. (2006). Pilot study of a self-administered stress management and exercise intervention during chemotherapy for cancer. Supportive Care in Cancer, 14, 928-935.
Wittgenstein, L. (1953). Philosophical investigations. New York: McMillan.

Yalom, I. D. (1985). The theory and practice of group psychotherapy. New York: W.W. Norton.

Yoo, H. J., Ahn, S. H., Kim, S. B., Kim, W. K., \& Han, O. S. (2005). Efficacy of progressive muscle relaxation training and guided imagery in reducing chemotherapy side effects in patients with breast cancer and in improving their quality of life. Supportive Care in Cancer, 13, 826-833. 
